\title{
$\beta$-Ketophosphonates as substrates in the Biginelli multicomponent reaction: an efficient and straightforward synthesis of phosphorylated dihydropyrimidinones
}

\author{
Idris Essid and Soufiane Touil* \\ Laboratory of Heteroatom Organic Chemistry, Department of Chemistry, \\ Faculty of Sciences of Bizerta, University of Carthage, 7021-Jarzouna, Tunisia \\ E-mail: soufiane.touil@fsb.rnu.tn
}

\begin{abstract}
Herein, we report an efficient and straightforward multicomponent synthesis of phosphorylated dihydropyrimidinone derivatives, via the $p$-toluenesulfonic acid promoted Biginelli-type reaction of $\beta$-ketophosphonates, aldehydes and urea. This method offers significant advantages over prior reports, such as efficiency, high yields and mild reaction conditions. Furthermore it is a green protocol avoiding hazardous metal-based catalysts.
\end{abstract}

Keywords: Multicomponent reactions; Biginelli reaction; dihydropyrimidinones; $\beta$ ketophosphonates; phosphorylated heterocycles

\section{Introduction}

Increasing interest has been devoted to multicomponent reactions as reliable synthetic methods. ${ }^{1-}$ 6 Such interest has been stimulated by their promising applications in combinatorial and medicinal chemistry. A multicomponent reaction involves three or more reagents reacting in a single step to form a new product which contains the essential parts of all the starting materials. This reaction tool allows compounds to be synthesized in a one-pot operation and with a simple purification process as all the reactants are incorporated into the final product.

The Biginelli reaction ${ }^{7-12}$ is an easy and useful three-component synthesis which involves the condensation of an aldehyde, urea, and a $\beta$-ketoester, under acid catalysis, to give 3,4dihydropyrimidinone derivatives (Scheme 1). These Biginelli adducts are associated with a wide range of biological properties including antimicrobial, ${ }^{13}$ antiviral, ${ }^{14}$ anti-inflammatory ${ }^{15-16}$ and anticancer ${ }^{17}$ activities; some are also medicinally important as calcium channel modulators. ${ }^{13}$ 


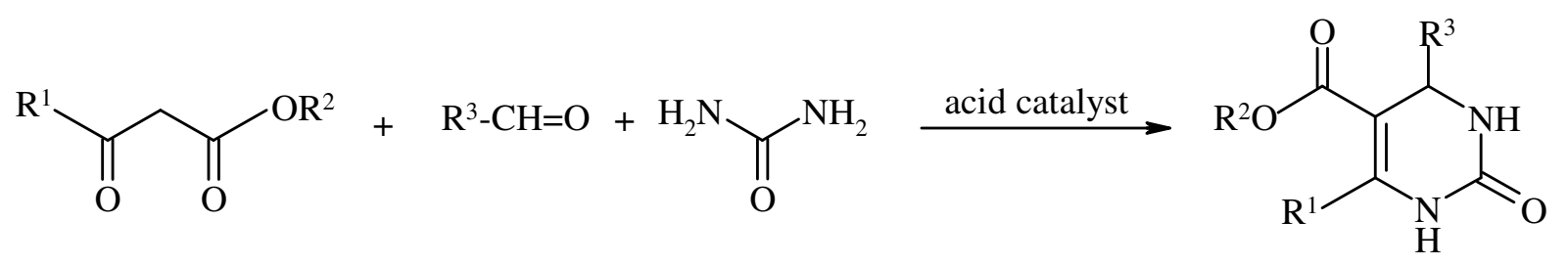

Scheme 1. The Biginelli reaction.

With this in mind, and in continuation of our research on the preparation of new phosphorylated heterocycles with possible biological properties, ${ }^{18-20}$ we have investigated the behaviour of $\beta$-ketophosphonates in the Biginelli reaction, which could represent an easy and direct access to novel types of 3,4-dihydropyrimidinone derivatives bearing a phosphonate group. The phosphorus substituent might regulate important biological functions and could improve the biological activity of such compounds, in a similar way to that reported for other pharmaceuticals. $^{21-25}$

To the best of our knowledge, there is only one report ${ }^{26}$ on the use of $\beta$ ketophosphonates as substrates in the Biginelli reaction which employed ytterbium triflate as catalyst in refluxing toluene. However, the desired phosphonatodihydropyrimidinones were obtained in low to moderate yields (15-58\%).

We thus wish to disclose the development of a new protocol for the synthesis of 5phosphonato-3,4-dihydropyrimidin-2-ones giving high yields and avoiding hazardous metalbased catalysts.

\section{Results and Discussion}

To optimize the reaction conditions for the formation of the target compounds, we used $\beta$ ketophosphonate 1a, benzaldehyde and urea as model substrates. The reaction was initially studied with various acid catalysts. The results of these comparative experiments are summarized in Table 1. It was found that performing the model reaction using inorganic acids such as $\mathrm{HCl}$ and $\mathrm{H}_{2} \mathrm{SO}_{4}$, or Lewis acids such as $\mathrm{SnCl}_{2}, \mathrm{FeCl}_{3}$ and $\mathrm{VCl}_{3}$, did not succeed; lower than $10 \%$ yield was obtained after refluxing the mixture in $\mathrm{MeCN}$ for long periods of time (Table 1, entries 1-5). Also tested was the use of heterogeneous catalysts such as silica gel supported sulfuric acid and sodium hydrogen sulfate, but this left the starting materials intact (Table 1, entries 6 and 7). An improvement in the yield was observed when using $20 \mathrm{~mol} \%$ of organic acid catalysts such as $\mathrm{AcOH}$ and $\mathrm{TsOH}$, with TsOH giving the best results (Table 1, entries 8 and 9). Under the same reaction conditions, it was gratifying to observe that $90 \%$ yield of the desired product 3a was obtained when the amount of $\mathrm{TsOH}$ was increased to $50 \mathrm{~mol} \%$ (Table 1, entry 10). 
Table 1. Optimization of the reaction conditions using different catalysts<smiles>CCOP(=O)(CC(C)=O)CC(C)=O</smiles>
1a $\mathbf{2 a}$<smiles>CCO[PH](=O)C1=C(C)NC(=O)NC1c1ccccc1</smiles>

3a

\begin{tabular}{|c|c|c|c|}
\hline Entry & Catalyst (mol\%) & Time (h) & Yield $(\%)^{a}$ \\
\hline 1 & $\mathrm{HCl}(20)$ & 72 & $0^{\mathrm{b}}$ \\
\hline 2 & $\mathrm{H}_{2} \mathrm{SO}_{4}(20)$ & 72 & $0^{\mathrm{b}}$ \\
\hline 3 & $\mathrm{SnCl}_{2} / \mathrm{HCl}(20)$ & 72 & 0 \\
\hline 4 & $\mathrm{FeCl}_{3} / \mathrm{HCl}(20)$ & 72 & 0 \\
\hline 5 & $\mathrm{VCl}_{3} / \mathrm{HCl}(20)$ & 72 & 10 \\
\hline 6 & $\mathrm{SiO}_{2}-\mathrm{H}_{2} \mathrm{SO}_{4}(20)$ & 72 & 0 \\
\hline 7 & $\mathrm{SiO}_{2}-\mathrm{NaHSO}_{4}(20)$ & 72 & 0 \\
\hline 8 & $\mathrm{AcOH}(20)$ & 72 & $20^{b}$ \\
\hline 9 & $\mathrm{TsOH}(20)$ & 72 & 50 \\
\hline 10 & $\mathrm{TsOH}(50)$ & 24 & 90 \\
\hline
\end{tabular}

${ }^{a}$ Isolated yield. ${ }^{b}$ No product was obtained when the reaction was carried out in EtOH as solvent.

The promising results obtained with $\mathrm{TsOH}(50 \mathrm{~mol} \%)$ as the catalyst prompted us to further investigate the effect of solvents on the reaction yield. As shown in Table 2, it was apparent that the reaction proceeded better in aprotic solvents compared to protic solvents. The best results were recorded with $\mathrm{MeCN}$ which gave a $90 \%$ yield of $3 \mathbf{a}$ (Table 2, entry 1).

Table 2. Optimization of the reaction conditions using different solvents

\begin{tabular}{|c|c|c|c|}
\hline \multirow[t]{2}{*}{$1 \mathbf{a}$} & \multirow{2}{*}{$+\quad \mathbf{2 a}$} & \multirow{2}{*}{$\prod_{\mathrm{O}}^{\mathrm{H}_{2} \mathrm{~N}}$} & \multirow{2}{*}{$\frac{\mathrm{TsOH}(50 \mathrm{~mol} \%)}{\text { solvent, reflux, } 24 \mathrm{~h}}$} \\
\hline & & & \\
\hline Entry & Solvent & Yield $(\%)^{a}$ & \\
\hline 1 & $\mathrm{MeCN}$ & 90 & \\
\hline 2 & 1,4-dioxane & 20 & \\
\hline 3 & $\mathrm{THF}$ & 15 & \\
\hline 4 & toluene & 40 & \\
\hline 5 & $\mathrm{EtOH}$ & 0 & \\
\hline 6 & $\mathrm{MeOH}$ & 0 & \\
\hline 7 & $\mathrm{H}_{2} \mathrm{O}$ & 0 & \\
\hline
\end{tabular}


With optimized reaction conditions in hand, we next studied the scope of this methodology. A variety of structurally diverse aldehydes and $\beta$-ketophosphonates were investigated and a series of 5-phosphonato-3,4-dihydropyrimidin-2-ones of type $\mathbf{3}$ were afforded in good yields (Table 3). The reactions proceeded efficiently with aromatic aldehydes bearing electronwithdrawing or electron-donating groups. However, aliphatic aldehydes were unreactive.

Although no intermediates were isolated or identified, we assume that formation of the phosphonatodihydropyrimidinones $\mathbf{3}$ followed the commonly accepted mechanism of the Biginelli condensation. ${ }^{7-12}$ Thus the reaction is thought to proceed via nucleophilic attack of urea on the aldehyde giving rise to an iminium intermediate. The interception of this last one by the $\beta$ ketophosphonate through its enol tautomer leads, after intramolecular cyclization and dehydration, to the dihydropyrimidinone 3 (Scheme 2).

Table 3. Substrate scope studies<smiles>[Y4]C(=O)C[PH]([R10])=O</smiles>

1<smiles>[R]C=[OH+]</smiles>

2<smiles>[R]C1NC(=O)NC(C)=C1P(=O)=O</smiles>

3

\begin{tabular}{ccccc}
\hline Entry & $\mathrm{R}^{1}$ & $\mathrm{R}^{2}$ & Product & Yield (\%) \\
\hline 1 & $\mathrm{Et}$ & $\mathrm{C}_{6} \mathrm{H}_{5}$ & 3a & 90 \\
2 & $\mathrm{Me}$ & $\mathrm{C}_{6} \mathrm{H}_{5}$ & $\mathbf{3 b}$ & 83 \\
3 & $\mathrm{Et}$ & $4-\mathrm{O}_{2} \mathrm{~N}^{a} \mathrm{C}_{6} \mathrm{H}_{4}$ & $\mathbf{3 c}$ & 96 \\
4 & $\mathrm{Me}$ & $4-\mathrm{O}_{2} \mathrm{~N}^{-} \mathrm{C}_{6} \mathrm{H}_{4}$ & $\mathbf{3 d}$ & 92 \\
5 & $\mathrm{Et}$ & $4-\mathrm{Cl}^{-} \mathrm{C}_{6} \mathrm{H}_{4}$ & $\mathbf{3 e}$ & 90 \\
6 & $\mathrm{Me}$ & $4-\mathrm{Cl}_{6} \mathrm{C}_{6} \mathrm{H}_{4}$ & $\mathbf{3 f}$ & 87 \\
7 & $\mathrm{Et}$ & $4-\mathrm{CH}_{3} \mathrm{O}-\mathrm{C}_{6} \mathrm{H}_{4}$ & $\mathbf{3 g}$ & 78 \\
8 & $\mathrm{Me}$ & $4-\mathrm{CH}_{3} \mathrm{O}-\mathrm{C}_{6} \mathrm{H}_{4}$ & $\mathbf{3 h}$ & 72 \\
\hline
\end{tabular}

${ }^{a}$ Isolated yield. 
<smiles>[R]C=[NH+]C(N)=O</smiles>

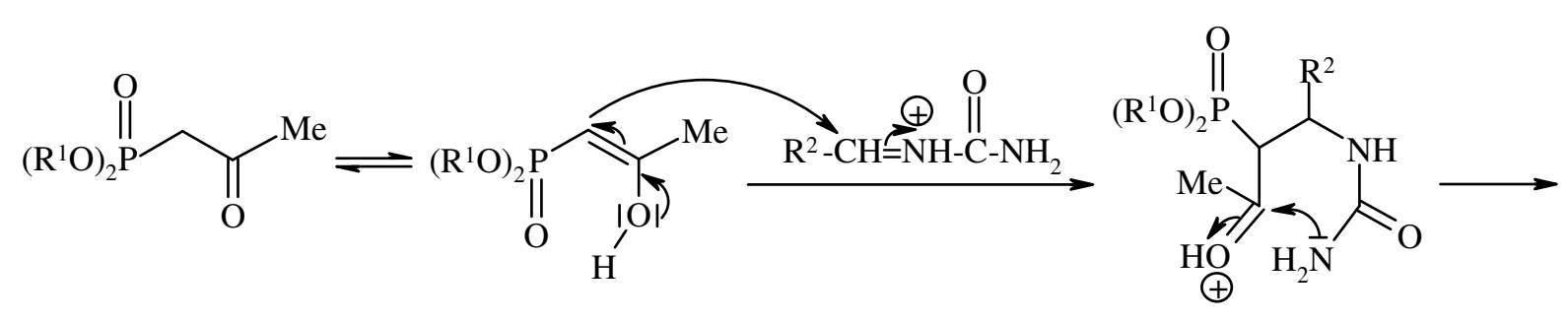<smiles>[R]C1NC(=O)NC([Y4])(O)C1P=O</smiles><smiles>[R]C1NC(=O)NC(C)=C1P(=O)=O</smiles>

3

Scheme 2. Reaction mechanism for the synthesis of compounds 3.

Products $\mathbf{3 b}$ and $\mathbf{3 d}$ are new compounds and their structures were established by examination of their IR, ${ }^{1} \mathrm{H},{ }^{13} \mathrm{C},{ }^{31} \mathrm{P}$ NMR and high resolution mass measurement of their molecular ions, whereas compounds $\mathbf{3 a}, \mathbf{3 c}$ and $\mathbf{3 e - 3 \mathbf { h }}$ have been identified by comparison of their IR and ${ }^{1} \mathrm{H}$ NMR spectroscopic data with the literature data. ${ }^{26}$ Furthermore, ${ }^{13} \mathrm{C}$ and ${ }^{31} \mathrm{P}$ NMR have been performed on derivatives 3a, 3c and 3e-3h. All spectroscopic data match the expected products. The ${ }^{31} \mathrm{P}$ NMR shifts recorded for compounds 3 occurred in the range 19-23 ppm, which is consistent with dialkylphosphonate chemical shift values. ${ }^{27}$

\section{Conclusions}

In summary, we have successfully developed an efficient and straightforward multicomponent synthesis of 5-phosphonato-3,4-dihydropyrimidin-2-ones, via the $p$-toluenesulfonic acid promoted Biginelli-type reaction of $\beta$-ketophosphonates, aldehydes and urea. By comparison with the existing strategy, ${ }^{26}$ our method offers significant advantages such as efficiency, high yields and mild reaction conditions. Furthermore it is a greener protocol avoiding hazardous metal-based catalysts. This is very beneficial for safely obtaining dihydropyrimidinone derivatives of pharmacological interest. 


\section{Experimental Section}

General. ${ }^{1} \mathrm{H},{ }^{31} \mathrm{P}$ and ${ }^{13} \mathrm{C}$ NMR spectra were recorded with DMSO- $d_{6}$ as the solvent, on a Bruker-300 spectrometer. The chemical shifts are reported in ppm relative to TMS (internal reference) for ${ }^{1} \mathrm{H}$ and ${ }^{13} \mathrm{C}$ NMR and relative to $85 \% \mathrm{H}_{3} \mathrm{PO}_{4}$ (external reference) for ${ }^{31} \mathrm{P} \mathrm{NMR}$. The coupling constants are reported in Hz. For the ${ }^{1} \mathrm{H}$ NMR, the multiplicities of signals are indicated by the following abbreviations: s: singlet, d: doublet, t: triplet, q: quartet, quint: quintet, m: multiplet. Mass spectra were determined on an Agilent 5975B spectrometer, under electronic impact (EI) conditions. IR spectra were recorded on a Nicolet IR200 spectrometer. The progress of the reactions was monitored by TLC. Purification of products was performed by column chromatography using silica gel 60 (Fluka).

\section{Synthesis of $\boldsymbol{\beta}$-ketophosphonates 1}

The starting $\beta$-ketophosphonates 1 were prepared according to reported procedures. ${ }^{28-29}$

General procedure for the synthesis of 5-phosphono-3,4-dihydropyrimidin-2-ones 3. A mixture of $\beta$-ketophosphonate $1(10 \mathrm{mmol})$, aldehyde $(10 \mathrm{mmol})$, urea $(15 \mathrm{mmol})$ and TsOH $(5$ $\mathrm{mmol})$ in dry MeCN $(10 \mathrm{~mL})$ was heated under reflux with stirring for $24 \mathrm{~h}$ (reactions were monitored by TLC). After cooling, the solvent was removed under reduced pressure and then $\mathrm{CHCl}_{3}(100 \mathrm{~mL})$ was added. The organic phase was extracted with $\mathrm{H}_{2} \mathrm{O}(2 \times 50 \mathrm{~mL})$, dried over $\mathrm{Na}_{2} \mathrm{SO}_{4}$ and concentrated under vacuum. The obtained residue was chromatographed on a silica gel column using EtOAc as eluent.

5-Diethoxyphosphoryl-6-methyl-4-phenyl-3,4-dihydropyrimidin-2(1H)-one $\quad(3 \mathrm{a}){ }^{26} \quad$ White solid; mp 169-170 ${ }^{\circ} \mathrm{C}$ (reported: $\left.168-170{ }^{\circ} \mathrm{C}\right) ;{ }^{31} \mathrm{P}\left\{{ }^{1} \mathrm{H}\right\}$ NMR (121.5 MHz, DMSO- $\left.d_{6}\right): \delta 19.1$;

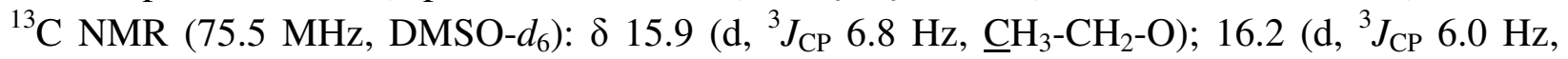
$\left.\mathrm{CH}_{3}-\mathrm{CH}_{2}-\mathrm{O}\right) ; 17.9$ (s, $\left.\mathrm{CH}_{3}\right) ; 56.4\left(\mathrm{~d},{ }^{2} J_{\mathrm{CP}} 15.8 \mathrm{~Hz}, \mathrm{CH}-\mathrm{NH}\right) ; 61.2\left(\mathrm{~d},{ }^{2} J_{\mathrm{CP}} 3.0 \mathrm{~Hz}, \mathrm{CH}_{3}-\underline{C H}_{2}-\mathrm{O}\right)$; $61.3\left(\mathrm{~d},{ }^{2} J_{\mathrm{CP}} 3.0 \mathrm{~Hz}, \mathrm{CH}_{3}-\underline{\mathrm{CH}}_{2}-\mathrm{O}\right) ; 95.6\left(\mathrm{~d},{ }^{1} J_{\mathrm{CP}} 207.6 \mathrm{~Hz}, \mathrm{P}-\underline{\mathrm{C}}=\mathrm{C}\right) ; 147.1$ (d, ${ }^{2} J_{\mathrm{CP}} 20.3 \mathrm{~Hz}, \mathrm{P}-$ $\mathrm{C}=\mathrm{C}) ; 154.5$ (s, $\mathrm{C}=\mathrm{O})$; phenyl carbons: $\delta$ 126.0, 127.3, 128.8, 143.8.

5-Dimethoxyphosphoryl-6-methyl-4-phenyl-3,4-dihydropyrimidin-2(1H)-one (3b). Yellow solid; mp 198-200 ${ }^{\circ} \mathrm{C} ;{ }^{31} \mathrm{P}\left\{{ }^{1} \mathrm{H}\right\}$ NMR (121.5 MHz, DMSO- $\left.d_{6}\right): \delta 22.8 ;{ }^{1} \mathrm{H}$ NMR $(300 \mathrm{MHz}$, DMSO-d $\left.d_{6}\right): \delta 2.09\left(\mathrm{~d}, 3 \mathrm{H},{ }^{4} J_{\mathrm{HP}} 3.0 \mathrm{~Hz}, \mathrm{CH}_{3}\right) ; 3.28\left(\mathrm{~d}, 3 \mathrm{H},{ }^{3} J_{\mathrm{PH}} 12.0 \mathrm{~Hz}, \mathrm{CH}_{3}-\mathrm{O}\right) ; 3.39\left(\mathrm{~d},{ }^{3} J_{\mathrm{PH}}\right.$ $\left.12.0 \mathrm{~Hz}, \mathrm{CH}_{3}-\mathrm{O}\right) ; 4.83\left(\mathrm{dd}, 1 \mathrm{H},{ }^{3} J_{\mathrm{PH}} 9.0 \mathrm{~Hz},{ }^{3} J_{\mathrm{HH}} 3.0 \mathrm{~Hz}, \mathrm{CH}-\mathrm{NH}\right) ; 7.08-7.47$ (m, 5H, arom-H); $7.69\left(\mathrm{~d}, 1 \mathrm{H},{ }^{3} J_{\mathrm{HH}} 3.0 \mathrm{~Hz}, \mathrm{~N}-\mathrm{H}\right) ; 9.19\left(\mathrm{~d}, 1 \mathrm{H},{ }^{4} \mathrm{~J}_{\mathrm{PH}} 3.0 \mathrm{~Hz}, \mathrm{~N}-\mathrm{H}\right) ;{ }^{13} \mathrm{C}$ NMR $(75.5 \mathrm{MHz}$, DMSO$\left.d_{6}\right): \delta 17.3\left(\mathrm{~d},{ }^{4} J_{\mathrm{CP}} 3.7 \mathrm{~Hz}, \mathrm{CH}_{3}\right) ; 51.2\left(\mathrm{~d},{ }^{2} J_{\mathrm{CP}} 4.5 \mathrm{~Hz}, \mathrm{CH}_{3}-\mathrm{O}\right) ; 51.4\left(\mathrm{~d},{ }^{2} J_{\mathrm{CP}} 5.2 \mathrm{~Hz}, \mathrm{CH}_{3}-\mathrm{O}\right)$; $54.9\left(\mathrm{~d},{ }^{2} J_{\mathrm{CP}} 15.1 \mathrm{~Hz}, \mathrm{CH}-\mathrm{NH}\right) ; 92.5\left(\mathrm{~d},{ }^{1} J_{\mathrm{CP}} 206.1 \mathrm{~Hz}, \mathrm{P}-\underline{\mathrm{C}}=\mathrm{C}\right) ; 148.7$ (d, $\left.{ }^{2} J_{\mathrm{CP}} 21.1 \mathrm{~Hz}, \mathrm{P}-\mathrm{C}=\underline{\mathrm{C}}\right)$; 152.4 (s, C=O); phenyl carbons: 126.6, 127.3, 128.3, 144.4; IR (neat): $v_{\mathrm{P}=\mathrm{O}} 1236 \mathrm{~cm}^{-1} ; v_{\mathrm{C}=\mathrm{O}} 1698$ $\mathrm{cm}^{-1} ; v_{\mathrm{NH}}$ 3275-3419 $\mathrm{cm}^{-1}$; EI-HRMS: calculated for $\mathrm{C}_{13} \mathrm{H}_{17} \mathrm{~N}_{2} \mathrm{O}_{4} \mathrm{P}: 296.0926\left(\mathrm{M}^{+}\right)$; found: 296.0922 .

5-Diethoxyphosphoryl-6-methyl-4-(4-nitrophenyl)-3,4-dihydropyrimidin-2(1H)-one $\quad(3 c){ }^{26}$ Clear yellow solid; mp 218-220 ${ }^{\circ} \mathrm{C}$ (reported: $\left.219-220{ }^{\circ} \mathrm{C}\right) ;{ }^{31} \mathrm{P}\left\{{ }^{1} \mathrm{H}\right\}$ NMR (121.5 MHz, DMSO-

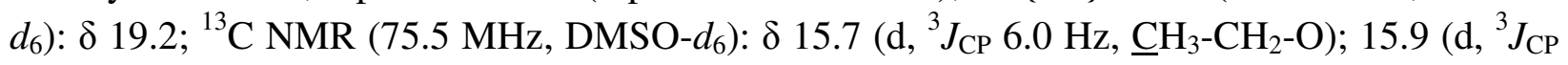


$\left.6.0 \mathrm{~Hz}, \mathrm{CH}_{3}-\mathrm{CH}_{2}-\mathrm{O}\right) ; 17.3\left(\mathrm{~s}, \mathrm{CH}_{3}\right) ; 54.5\left(\mathrm{~d},{ }^{2} J_{\mathrm{CP}} 15.8 \mathrm{~Hz}, \mathrm{CH}-\mathrm{NH}\right) ; 60.6\left(\mathrm{~d},{ }^{2} J_{\mathrm{CP}} 4.5 \mathrm{~Hz}, \mathrm{CH}_{3^{-}}\right.$ $\underline{\mathrm{CH}}_{2}-\mathrm{O}$ ); 60.7 (d, $\left.{ }^{2} J_{\mathrm{CP}} 5.2 \mathrm{~Hz}, \mathrm{CH}_{3}-\underline{C H}_{2}-\mathrm{O}\right) ; 92.7$ (d, $\left.{ }^{1} J_{\mathrm{CP}} 206.7 \mathrm{~Hz}, \mathrm{P}-\underline{\mathrm{C}}=\mathrm{C}\right) ; 149.0\left(\mathrm{~d},{ }^{2} J_{\mathrm{CP}} 21.0\right.$ $\mathrm{Hz}, \mathrm{P}-\mathrm{C}=\mathrm{C}) ; 152.8$ (s, C=O); phenyl carbons: 123.6, 127.8, 146.7, 151.5.

5-Dimethoxyphosphoryl-6-methyl-4-(4-nitrophenyl)-3,4-dihydropyrimidin-2(1H)-one (3d). Yellow solid; mp 235-237 ${ }^{\circ} \mathrm{C} ;{ }^{31} \mathrm{P}\left\{{ }^{1} \mathrm{H}\right\}$ NMR (121.5 MHz, DMSO-d $\left.d_{6}\right): \delta 22.3 ;{ }^{1} \mathrm{H}$ NMR (300 MHz, DMSO- $\left.d_{6}\right): \delta 2.08\left(\mathrm{~d}, 3 \mathrm{H},{ }^{4} J_{\mathrm{PH}} 3.0 \mathrm{~Hz}, \mathrm{CH}_{3}\right) ; 3.34\left(\mathrm{~d}, 3 \mathrm{H},{ }^{3} J_{\mathrm{PH}} 12.0 \mathrm{~Hz}, \mathrm{CH}_{3}-\mathrm{O}\right) ; 3.39(\mathrm{~d}$, $\left.3 \mathrm{H},{ }^{3} J_{\mathrm{PH}} 12.0 \mathrm{~Hz}, \mathrm{CH}_{3}-\mathrm{O}\right) ; 4.98\left(\mathrm{dd}, 1 \mathrm{H},{ }^{3} J_{\mathrm{PH}} 9.0 \mathrm{~Hz},{ }^{3} J_{\mathrm{HH}} 3.0 \mathrm{~Hz}, \mathrm{CH}-\mathrm{NH}\right) ; 7.52-8.23$ (m, 4H, arom-H); 7.85 (br s, $1 \mathrm{H}, \mathrm{N}-\mathrm{H}) ; 9.37\left(\mathrm{~d}, 1 \mathrm{H},{ }^{4} J_{\mathrm{PH}} 3.0 \mathrm{~Hz}, \mathrm{~N}-\mathrm{H}\right) ;{ }^{13} \mathrm{C}$ NMR $\left(75.5 \mathrm{MHz}\right.$, DMSO- $\left.d_{6}\right)$ : $\delta 17.3\left(\mathrm{~s}, \mathrm{CH}_{3}\right) ; 51.5\left(\mathrm{~d},{ }^{2} J_{\mathrm{CP}} 3.0 \mathrm{~Hz}, \mathrm{CH}_{3} \mathrm{O}\right) ; 51.5\left(\mathrm{~d},{ }^{2} J_{\mathrm{CP}} 3.7 \mathrm{~Hz}, \mathrm{CH}_{3}-\mathrm{O}\right) ; 54.5\left(\mathrm{~d},{ }^{2} J_{\mathrm{CP}} 15.1\right.$ $\mathrm{Hz}, \mathrm{CH}-\mathrm{NH}$ ); 91.4 (d, $\left.{ }^{1} J_{\mathrm{CP}} 206.8 \mathrm{~Hz}, \mathrm{P}-\underline{\mathrm{C}}=\mathrm{C}\right) ; 149.6$ (d, $\left.{ }^{2} J_{\mathrm{CP}} 21.1 \mathrm{~Hz}, \mathrm{P}-\mathrm{C}=\underline{\mathrm{C}}\right) ; 152.1$ (s, C=O); phenyl carbons: $123.7,127.7,146.7,151.5$; IR (neat): $v_{\mathrm{P}=\mathrm{O}} 1230 \mathrm{~cm}^{-1} ; v_{\mathrm{C}=\mathrm{O}} 1710 \mathrm{~cm}^{-1} ; v_{\mathrm{NH}} 3225-$ $3373 \mathrm{~cm}^{-1}$; EI-HRMS: calculated for $\mathrm{C}_{13} \mathrm{H}_{16} \mathrm{~N}_{3} \mathrm{O}_{6} \mathrm{P}: 341.0777\left(\mathrm{M}^{+}\right)$; found: 341.0776 .

5-Diethoxyphosphoryl-6-methyl-4-(4-chlorophenyl)-3,4-dihydropyrimidin-2(1H)-one (3e). ${ }^{26}$ Yellow solid; mp 120-122 ${ }^{\circ} \mathrm{C}$ (reported: $\left.118-119{ }^{\circ} \mathrm{C}\right) ;{ }^{31} \mathrm{P}\left\{{ }^{1} \mathrm{H}\right\}$ NMR (121.5 MHz, DMSO- $\left.d_{6}\right): \delta$ 19.8; ${ }^{13} \mathrm{C}$ NMR $\left(75.5 \mathrm{MHz}, \mathrm{DMSO}-d_{6}\right): \delta 15.6\left(\mathrm{~d},{ }^{3} J_{\mathrm{CP}} 7.5 \mathrm{~Hz}, \underline{\mathrm{CH}}_{3}-\mathrm{CH}_{2}-\mathrm{O}\right) ; 15.7\left(\mathrm{~d},{ }^{3} J_{\mathrm{CP}} 6.8\right.$ $\mathrm{Hz}, \mathrm{CH}_{3}-\mathrm{CH}_{2}-\mathrm{O}$ ); 17.2 (s, CH3); 54.4 (d, $\left.{ }^{2} J_{\mathrm{CP}} 15.8 \mathrm{~Hz}, \mathrm{CH}-\mathrm{NH}\right) ; 60.5$ (d, ${ }^{2} J_{\mathrm{CP}} 4.5 \mathrm{~Hz}, \mathrm{CH}_{3}-\underline{C H}_{2}-$ O); $60.6\left(\mathrm{~d},{ }^{2} J_{\mathrm{CP}} 3.8 \mathrm{~Hz}, \mathrm{CH}_{3}-\underline{C H}_{2}-\mathrm{O}\right) ; 95.7$ (d, $\left.{ }^{1} J_{\mathrm{CP}} 207.6 \mathrm{~Hz}, \mathrm{P}-\underline{\mathrm{C}}=\mathrm{C}\right) ; 148.4\left(\mathrm{~d},{ }^{2} J_{\mathrm{CP}} 20.4 \mathrm{~Hz}, \mathrm{P}-\right.$ $\mathrm{C}=\mathrm{C}) ; 152.8$ (s, $\mathrm{C}=\mathrm{O})$; phenyl carbons: 128.6, 131.6, 140.4, 143.4.

5-Dimethoxyphosphoryl-6-methyl-4-(4-chlorophenyl)-3,4-dihydropyrimidin-2(1H)-one (3f). ${ }^{26}$ Clear yellow solid; mp 226-227 ${ }^{\circ} \mathrm{C}$ (reported: $\left.229-230{ }^{\circ} \mathrm{C}\right) ;{ }^{31} \mathrm{P}\left\{{ }^{1} \mathrm{H}\right\} \mathrm{NMR}(121.5 \mathrm{MHz}$, DMSO- $\left.d_{6}\right): \delta 22.6 ;{ }^{13} \mathrm{C}$ NMR $\left(75.5 \mathrm{MHz}, \mathrm{DMSO}-d_{6}\right): \delta 17.3\left(\mathrm{~d},{ }^{4} J_{\mathrm{CP}} 3.7 \mathrm{~Hz}, \mathrm{CH}_{3}\right) ; 51.3\left(\mathrm{~d},{ }^{2} J_{\mathrm{CP}}\right.$ $\left.5.3 \mathrm{~Hz}, \mathrm{CH}_{3}-\mathrm{O}\right) ; 51.5\left(\mathrm{~d},{ }^{2} J_{\mathrm{CP}} 5.3 \mathrm{~Hz}, \mathrm{CH}_{3}-\mathrm{O}\right) ; 54.3\left(\mathrm{~d},{ }^{2} J_{\mathrm{CP}} 15.1 \mathrm{~Hz}, \mathrm{CH}-\mathrm{NH}\right) ; 92.2\left(\mathrm{~d},{ }^{1} J_{\mathrm{CP}}\right.$ $206.1 \mathrm{~Hz}, \mathrm{P}-\underline{\mathrm{C}}=\mathrm{C}) ; 149.0$ (d, ${ }^{2} J_{\mathrm{CP}} 21.1 \mathrm{~Hz}, \mathrm{P}-\mathrm{C}=\underline{\mathrm{C}}$ ); 152.3 (s, C=O); phenyl carbons: 128.3, 131.9, 140.0, 143.3.

\section{5-Diethoxyphosphoryl-6-methyl-4-(4-methoxyphenyl)-3,4-dihydropyrimidin-2(1H)-one} (3g). ${ }^{26}$ Clear yellow solid; mp 138-140 ${ }^{\circ} \mathrm{C}$ (reported: $\left.137-139{ }^{\circ} \mathrm{C}\right) ;{ }^{31} \mathrm{P}\left\{{ }^{1} \mathrm{H}\right\} \mathrm{NMR}(121.5 \mathrm{MHz}$, DMSO- $\left.d_{6}\right): \delta 19.5 ;{ }^{13} \mathrm{C}$ NMR (75.5 MHz, DMSO- $\left.d_{6}\right): \delta 15.9\left(\mathrm{~d},{ }^{3} J_{\mathrm{CP}} 6.0 \mathrm{~Hz}, \underline{\mathrm{CH}}_{3}-\mathrm{CH}_{2}-\mathrm{O}\right) ; 16.0$ $\left(\mathrm{d},{ }^{3} J_{\mathrm{CP}} 6.8 \mathrm{~Hz}, \underline{\mathrm{CH}}_{3}-\mathrm{CH}_{2}-\mathrm{O}\right) ; 54.3\left(\mathrm{~d},{ }^{2} J_{\mathrm{CP}} 15.1 \mathrm{~Hz}, \mathrm{CH}-\mathrm{NH}\right) ; 55.0\left(\mathrm{~s}, \mathrm{CH}_{3}-\mathrm{O}\right) ; 60.5$ (d, ${ }^{2} J_{\mathrm{CP}} 5.3$ $\left.\mathrm{Hz}, \mathrm{CH}_{3}-\underline{\mathrm{CH}}_{2}-\mathrm{O}\right) ; 61.0\left(\mathrm{~d},{ }^{2} J_{\mathrm{CP}} 6.0 \mathrm{~Hz}, \mathrm{CH}_{3}-\underline{C H}_{2}-\mathrm{O}\right) ; 95.2\left(\mathrm{~d},{ }^{1} J_{\mathrm{CP}} 206.7 \mathrm{~Hz}, \mathrm{P}-\underline{\mathrm{C}}=\mathrm{C}\right) ; 148.0(\mathrm{~d}$, $\left.{ }^{2} J_{\mathrm{CP}} 21.0 \mathrm{~Hz}, \mathrm{P}-\mathrm{C}=\underline{\mathrm{C}}\right) ; 153.9$ (s, C=O); phenyl carbons: 113.5, 127.3, 136.6 158.6.

\section{5-Dimethoxyphosphoryl-6-methyl-4-(4-methoxyphenyl)-3,4-dihydropyrimidin-2(1H)-one} (3h). ${ }^{26}$ Yellow solid; mp 214-216 ${ }^{\circ} \mathrm{C}$ (reported: 216-217 $\left.{ }^{\circ} \mathrm{C}\right) ;{ }^{31} \mathrm{P}\left\{{ }^{1} \mathrm{H}\right\}$ NMR $(121.5 \mathrm{MHz}$, DMSO- $\left.d_{6}\right): \delta 22.9 ;{ }^{13} \mathrm{C}$ NMR (75.5 MHz, DMSO-d $): \delta 17.2\left(\mathrm{~s}, \mathrm{CH}_{3}\right) ; 51.6\left(\mathrm{~d},{ }^{2} J_{\mathrm{CP}} 5.3 \mathrm{~Hz}, \mathrm{CH}_{3^{-}}\right.$ O-P); 51.6 (d, $\left.{ }^{2} J_{\mathrm{CP}} 5.3 \mathrm{~Hz}, \mathrm{CH}_{3}-\mathrm{O}-\mathrm{P}\right) ; 54.3$ (d, $\left.{ }^{2} J_{\mathrm{CP}} 15.1 \mathrm{~Hz}, \mathrm{CH}-\mathrm{NH}\right) ; 55.0$ (s, $\left.\mathrm{CH}_{3}-\mathrm{O}\right) ; 94.9$ (d, $\left.{ }^{1} J_{\mathrm{CP}} 206.8 \mathrm{~Hz}, \mathrm{P}-\underline{\mathrm{C}}=\mathrm{C}\right) ; 148.4\left(\mathrm{~d},{ }^{2} J_{\mathrm{CP}} 21.1 \mathrm{~Hz}, \mathrm{P}-\mathrm{C}=\underline{\mathrm{C}}\right) ; 155.0(\mathrm{~s}, \mathrm{C}=\mathrm{O})$; phenyl carbons: 114.2 , 127.7, 136.6, 158.6. 


\section{Acknowledgments}

We thank the Tunisian Ministry of Higher Education and Scientific Research for financial support.

\section{References}

1. Domling, A.; Ugi, I. Angew. Chem. Int. Ed. 2000, 39, 3168-3210. http://dx.doi.org/10.1002/1521-3773(20000915)39:18<3168::AID-ANIE3168>3.0.CO;2-U

2. Nair, V.; Rajesh, C.; Vinod, A. U.; Bindu, S.; Sreekenth, A. R.; Balagopal, L. Acc. Chem. Res. 2003, 36, 899-907. http://dx.doi.org/10.1021/ar020258p

PMid:14674781

3. Zhu, J. P.; Bienayme, H. Eds. Multicomponent Reactions; Wiley-VCH: Weinheim: Germany, 2005. http://dx.doi.org/10.1002/3527605118

4. Domling, A. Chem. Rev. 2006, 106, 17-89. http://dx.doi.org/10.1021/cr0505728 PMid:16402771

5. Tejedor, D.; Garcia-Tellado, F. Chem. Soc. Rev. 2007, 36, 484-491. http://dx.doi.org/10.1039/b608164a PMid:17325787

6. Sunderhaus, J. D.; Martin, S. F. Chem. Eur. J. 2009, 15, 1300-1308. http://dx.doi.org/10.1002/chem.200802140 PMid:19132705 PMCid:2803615

7. Kappe, C. O. Tetrahedron 1993, 49, 6937-6963. http://dx.doi.org/10.1016/S0040-4020(01)87971-0

8. Kappe, C. O. Acc. Chem. Res. 2000, 33, 879-888. http://dx.doi.org/10.1021/ar000048h PMid:11123887

9. Kappe, C. O.; Stadler, A. Org. React. 2004, 63, 1-116.

10. Vdovina, S. V.; Mamedov, V. A. Russ. Chem. Rev. 2008, 77, 1017-1053. http://dx.doi.org/10.1070/RC2008v077n12ABEH003894

11. Syamala, M. Org. Prep. Proced. Int. 2009, 41, 1-68. http://dx.doi.org/10.1080/00304940802711218

12. Suresh; Sandhu, J. S. Arkivoc 2012, (i), 66-133.

13. Kappe, C. O. Eur. J. Med. Chem. 2000, 35, 1043-1052. http://dx.doi.org/10.1016/S0223-5234(00)01189-2 
14. Hurst, E. W.; Hull, R. J. Med. Pharm. Chem. 1961, 3, 215-229. http://dx.doi.org/10.1021/jm50015a002

15. Tozkoparan, B.; Ertan, M.; Kelicen, P.; Demirdamar, R. Farmaco 1999, 54, 588-593. http://dx.doi.org/10.1016/S0014-827X(99)00068-3

16. Sadanandam, Y. S.; Shetty, M. M.; Dwan, P. V. Eur. J. Med. Chem. 1992, 27, 87-92. http://dx.doi.org/10.1016/0223-5234(92)90066-A

17. Wright, C. M.; Chovatiya, R. J.; Jameson, N. E.; Turner, D. M.; Zhu, G.; Werner, S.; Huryn, D. M.; Pipas, J. M.; Day, B. W.; Wipf, P.; Brodsky, J. L. Bioorg. Med. Chem. 2008, 16, 3291-3301.

http://dx.doi.org/10.1016/j.bmc.2007.12.014

PMid:18164205 PMCid:2330198

18. Chebil, E.; Chamakhi, M.; Touil, S. J. Sulfur Chem. 2011, 32, 249-256. http://dx.doi.org/10.1080/17415993.2011.570763

19. Ben Gaied, L.; Touil, S.; Zantour, H. Phosphorus, Sulfur Silicon Relat. Elem. 2006, 181, 601608.

http://dx.doi.org/10.1080/10426500500269844

20. Touil, S.; Zantour, H. Phosphorus, Sulfur Silicon Relat. Elem. 2003, 178, 353-360. http://dx.doi.org/10.1080/10426500307947

21. Palacios, F.; Alonso, C.; de los Santos, J. M. Chem. Rev. 2005, 105, 899-931. http://dx.doi.org/10.1021/cr040672y

PMid:15755080

22. Kafarski, P.; Lejezak, B. Phosphorus Sulfur 1991, 63, 193-215. http://dx.doi.org/10.1080/10426509108029443

23. Hoagland, R. E. Biologically Active Natural Products; Culter, H. G., Ed.; ACS Symposium Series 380. American Chemical Society: Washington DC, 1988; p 182.

24. Toy, A. D. F.; Walsh, E. N. In Phosphorus Chemistry in Everyday Living; American Chemical Society: Washington D. C., 1987.

PMCid:174885

25. Engel, R. In Handbook of Organophosphorus Chemistry; M. Dekker: New York, 1992.

26. Gong, D.; Zhang, L.; Yuan, C. Heteroatom Chem. 2003, 14, 13-17. http://dx.doi.org/10.1002/hc.10058

27. Tebby, J. C., Ed., Handbook of Phosphorus-31 Nuclear Magnetic Resonance Data, CRC Press: Boca Raton, FL, 1991.

28. Kitamura, M.; Tokunaga, M.; Noyori, R. J. Am. Chem. Soc. 1995, 117, 2931- 2932. http://dx.doi.org/10.1021/ja00115a030

29. Mathey, F.; Savignac, P. Tetrahedron 1978, 34, 649-654. http://dx.doi.org/10.1016/0040-4020(78)88099-5 